\title{
EGU21-9887
}

https://doi.org/10.5194/egusphere-egu21-9887

EGU General Assembly 2021

(c) Author(s) 2022. This work is distributed under

the Creative Commons Attribution 4.0 License.

\section{Assessing traditional irrigation systems in data scarcity conditions, a proposal for a methodological approach.}

\author{
Vladimiro Boselli ${ }^{1}$, Eleni Maria Michailidi ${ }^{2}$, Jalal Kassout ${ }^{3}$, Mhammad Houssni ${ }^{3}$, Abdeltif El \\ Ouahrani ${ }^{3}$, Matteo Sesana ${ }^{4}$, Massimiliano Borroni ${ }^{4}$, and Simone Cristoforetti ${ }^{4}$ \\ ${ }^{1}$ Università degli Studi di Brescia, DICATAM, Dipartimento di Ingegneria Civile, Architettura, Territorio, Ambiente e di \\ Matematica (v.boselli@unibs.it) \\ ${ }^{2}$ Direzione Difesa del Suolo, Regione del Veneto, (Italy) \\ ${ }^{3}$ Université Abdelmalek Essadi, Tétouan, Département de Biologie \\ ${ }^{4}$ Università Ca' Foscari Venezia, DSAAM - Dipartimento di Studi sull'Asia e sull'Africa Mediterranea
}

Traditional irrigation systems, apart from being an important cultural heritage element, are considered vital for sustainable water resource management and climate change adaptation measures. However, these traditional forms of irrigation and agriculture, with direct implications in food security at a local scale, have been suffering from abandonment or degradation worldwide. In light of this, the need to fully comprehend the complex linkage of their abandonment with different driving forces is essential. The identification of these dynamics enables the adoption of appropriate interventions with local initiatives and policies on a larger scale.

The present scientific contribution aims at presenting a valid methodology to consistently address the multidisciplinarity and the multifacetedness that emerge in studies relating to traditional irrigation systems.

The methodological approach introduced regards that of system dynamics and is geared to outline a combined framework at the service of stakeholders and policy makers. This approach has been already adopted previously, in different studies to tackle down the complexity stemming from the heterogeneity of drivers in complex water-related and ecosystem modeling problems. Among its advantages there are its ease of implementation in any given scenario and its ability to integrate qualitative and quantitative assessments of multidisciplinary nature that can even be interconnected. Moreover, its applicability in cases affected by data scarcity allows to address issues in those areas of the world which often are more vulnerable, poorer and marginalized and which consequently suffer from a lack of interest in monitoring environmental and social variables, properly.

The abstract is based on Boselli, V., Ouallali, A., Briak, H., Houssni, M., Kassout, J., El Ouahrani, A., \& Michailidi, E. M. (2020). System Dynamics Applied to Terraced Agroecosystems: The Case Study of Assaragh (Anti-Atlas Mountains, Morocco). Water, 12(6), 1693. doi.org/10.3390/w12061693 
